\title{
The relevance of balance assessment to detect balance deficits in obese subjects: A cross sectional study.
}

\author{
Ibrahim AA ${ }^{1^{*}}$, Mohamed AMR ${ }^{2}$, Kamel EM², Youssef RFM ${ }^{2}, A l-M a t h o u r ~ R S^{2}$ \\ ${ }^{1}$ Department of Physiotherapy, Cairo University Hospitals, Cairo, Egypt \\ ${ }^{2}$ Department of Physical Therapy, College of Applied Medical Sciences, University of Hail, Saudi Arabia
}

\begin{abstract}
Objectives: This study was conducted to assess posture balance and fall risk in obese men.

Design: A cross sectional study.

Methods: Fifty males were represented the sample of the study. We used Biodex balance system for assessing three tests for the subject i.e., Posture stability testing, Limits of stability testing and Fall risk testing.
\end{abstract}

Results: Descriptive statistics of means and standard deviations and correlation analysis using Pearson correlation coefficient revealed no statistical difference between tested parameters with change in BMI.

Conclusion: We concluded that BMI alone is not main cause of balance but other factors may have greater role in stability.

Keywords: Obesity, Balance, Stability, Sample.

\section{Introduction}

Obesity is abnormal fat accumulation in body tissue to the extent that health may be affected [1]. Obesity is becoming a worldwide problem affecting all levels of society and is being described as a global epidemic [2].

It has become an important health problem; it is the fifth leading cause of mortality worldwide. In addition, it is a risk factor for many diseases such as cancers, diabetes mellitus, metabolic syndrome, hypertension and cardiac disorders [3-7].

Obesity was defined as BMI more than or equal to 30. (BMI) Body mass index was evaluated as weight in kilograms divided by height in meters squared rounded to one decimal place [8].

Health professionals working in clinical specialties use term of Balance in a wide variety. There is no definition accepted of human balance, or related terms. Our study identifies definitions of balance and introduces clinical definitions of balance and postural control. Postural control is defined as the maintaining or achieving the state of balance during any activity. Postural control strategies may be predictive or reactive, and may involve either a fixed-support or a change-in-support response. Clinical examination of balance evaluates all components of balance ability. Health professionals must select his clinical assessments based on a true knowledge and understanding the classification of balance and postural control [9].

One fundamental property of postural equilibrium is stability. Postural stability refers to persistent motor control and an unchanged erect posture in response to a disturbance, or fluctuations caused by a disturbance. Generally, posture is considered stable if, when disturbed, it returns to its original state. Speed plays part instability.
The faster the posture returns to its original state, the more stable the posture is considered. Both, the resistance and fast equilibrium recovery are strongly dependent on body mass [10]. Postural control is any act of maintaining or restoring balance in static and dynamic posture. Balance is the state of equilibrium and the sum of forces, acting on the body is zero [11].

Balance is defined as, the ability to maintain the body's center of gravity (COG) over its base of support (BOS). Good balance exists when multiple systems (e.g. visual, vestibular, sensory and motor systems) interact automatically, providing accurate and exact information to the nervous system [12].

The major predisposing cause is diabetic polyneuropathy because the sensory In addition to increase in mortality, obese subjects face a greater risk of impairments. Obesity is a risk factor for functional deterioration in both genders, and the risk increases with body mass $[13,14]$.

There are a number of studies with obese boys providing support to this association between body weight and balance stability $[15,16]$.

Therefore, the scope of our study was to assessment of posture balance and fall risk of obesity in males in the University of Hail, KSA.

\section{Materials and Methods}

\section{Materials}

Biodex balance system: Balance ability was measured with the Biodex Balance System (BBS) (Model 302-945; Shirley, New York, USA). 


\section{Methods}

1. The postural stability test: Test the patient's ability to maintain center of balance.

2. The limit of stability test: Test the patients how to move and control his center of gravity within their base of support.

3. Performing a fall risk test: The fall risk test allows identification of potential fall candidates.

\section{Statistical Analysis}

Statistical analysis was performed on the data obtained from 50 subjects. Statistical analysis was performed using SPSS for Windows 18.0; measuring (frequency and percentage and correlation) with Pearson correlation coefficient test.

\section{Results}

The results of this study revealed the descriptive statistics of all parameters studied and the correlation between BMI and other parameters. the frequency distribution of, overall stability index with mean $2.27 \pm 1.056$ and Pearson correlation is 0.62 , limits of stability (overall) with mean $47.08 \pm 13.54$ and Pearson correlation is 0.57 , limits of stability (forward) with mean $58.72 \pm 15.305$ and Pearson correlation is -0.71 , limits of stability (backward) with mean $58.52 \pm 20.617$ and Pearson correlation is 0.46 , limits of stability (left) with mean $60.54 \pm 16.511$ and Pearson correlation is 0.183 , limits of stability (right) with mean $54.32 \pm 17.242$ and Pearson correlation is -0.009 , limits of stability (forward left) with mean $54.24 \pm 16.053$ and Pearson correlation is 0.195 , limits of stability (forward right) with mean $53.7 \pm 19.173$ and Pearson correlation is 0.12 , limits of stability (backward left) with mean $50.74 \pm 17.294$ and Pearson correlation is 0.27 and limits of stability (backward right) with mean 52.56 \pm 18.285 and Pearson correlation is 0.78 (Figures 1-7 and Tables 1 and 2).

\section{Discussion}

The present study focused in assessment of balance and risk of fall in obese males as a trial to know all the possible bad consequences of obesity. In addition to descriptive statistics the present study tried to make a correlation test between BMI and determents of balance. Biodex balance system is used in this study and this is on the contrary of Kumaresan and Kavithayini who used star excursion balance test in assessment of balance. Our results comes in disagreement with Jose Aliton who reported decreased stability limits in obese elderly men and this may be because of old age which induce decreased strength which makes obesity more effective in losing ability to resist falls. These results may be due to the sample we chosen was young students with good strength so they can resist the bad effects of obesity on balance but with increasing age deterioration of strength makes obesity a great determent in losing balance and increasing fall risk.

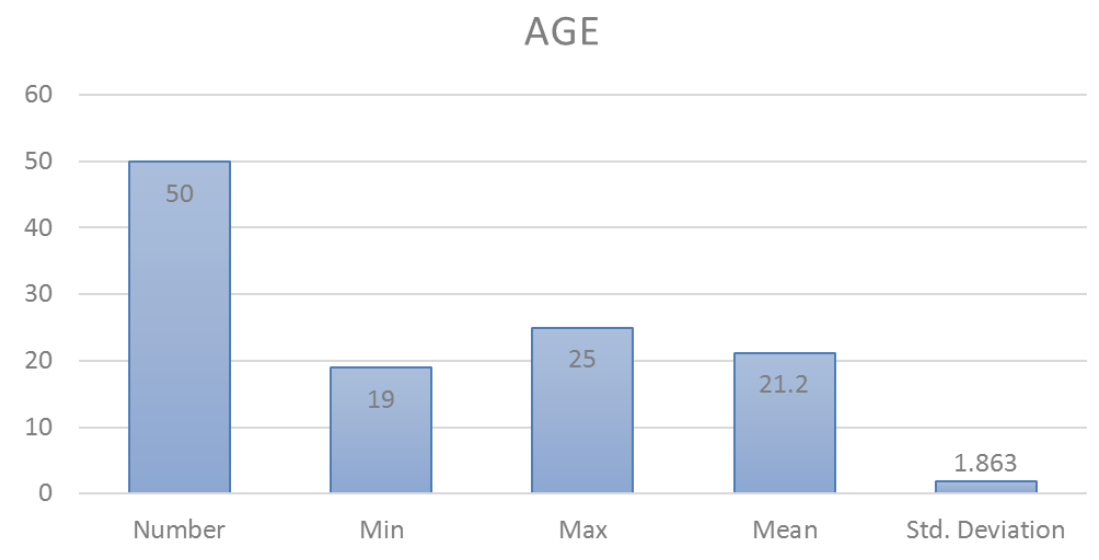

Figure 1. The frequency distribution of age with the mean is $21.2 \pm 1.863$.

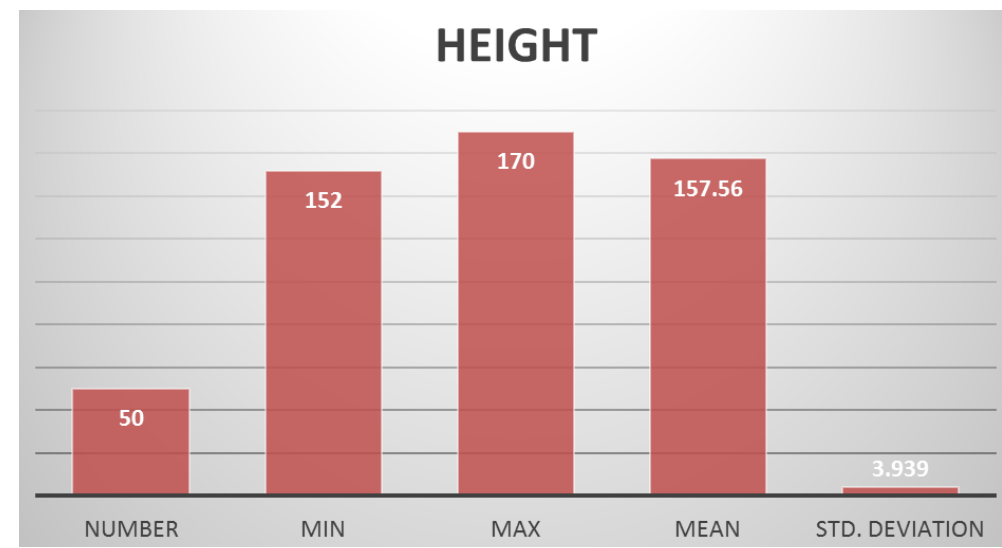

Figure 2. The frequency distribution of height with mean is $157.56 \pm 3.939$. 


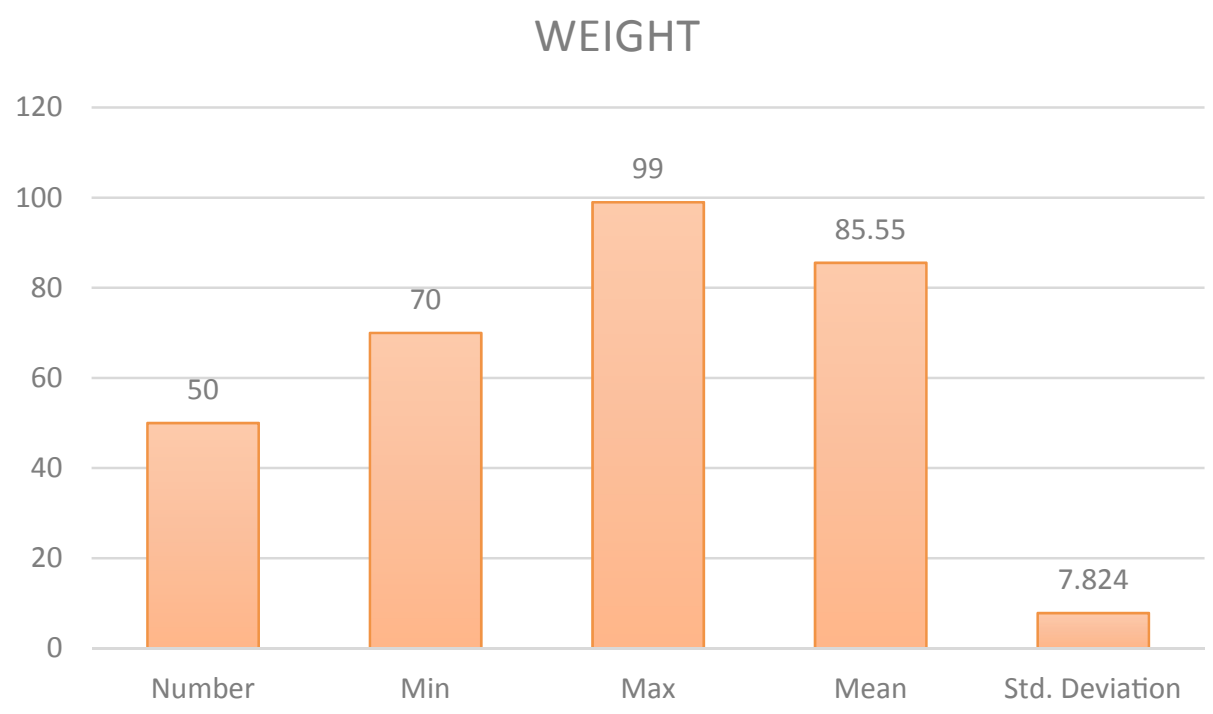

Figure 3. The frequency distribution of weight with mean is $85.55 \pm 7.824$.

\section{BMI}

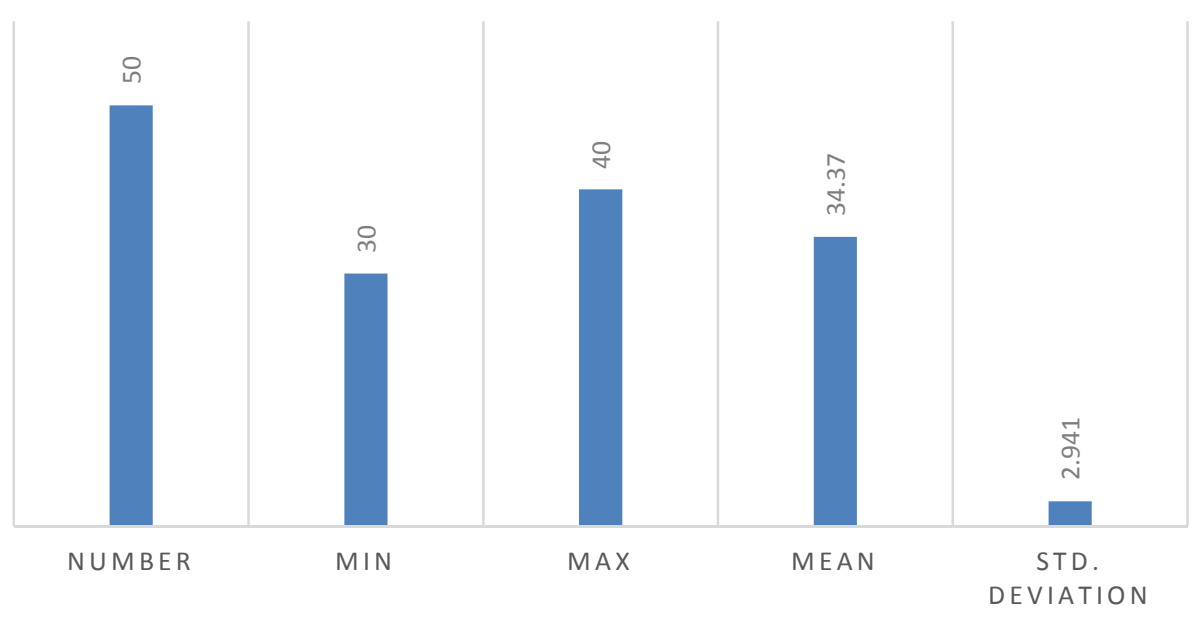

Figure 4. The frequency distribution of BMI with mean $34.37 \pm 2.941$.

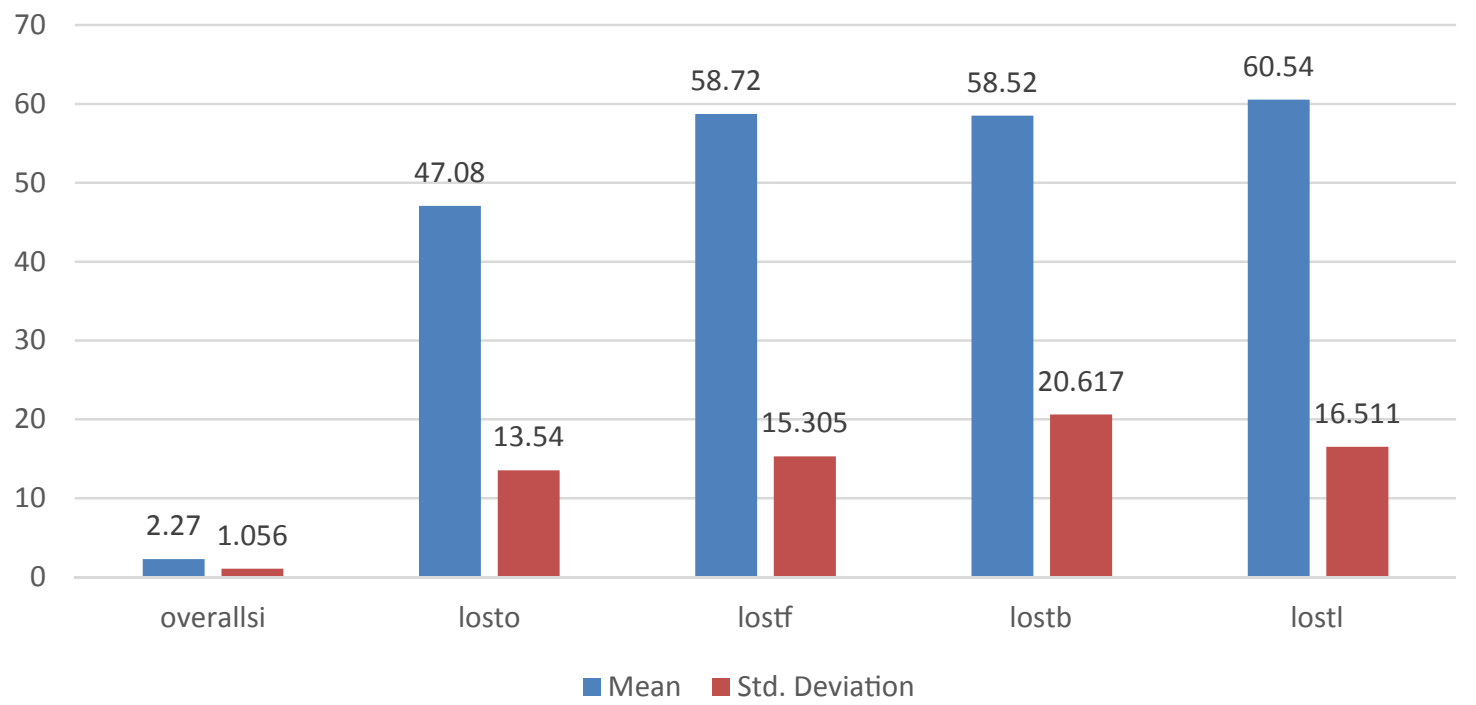

Figure 5. The frequency distribution of, overall stability(OVERALLSI) index with mean $2.27 \pm 1.056$, limits of stability (LOSTO) with mean 47.08 \pm 13.54 , limits of stability forward (LOSTF) with mean $58.72 \pm 15.305$, limits of stability backward (LOSTB)with mean $58.52 \pm 20.617$ and limits of stability left (LOSTL) with mean $60.54 \pm 16.511$. 
Citation: Ibrahim AA, Mohamed AMR, Kamel EM, et al. The relevance of balance assessment to detect balance deficits in obese subjects: A cross sectional study. Biol Med Case Rep. 2018;2(1):15-20.

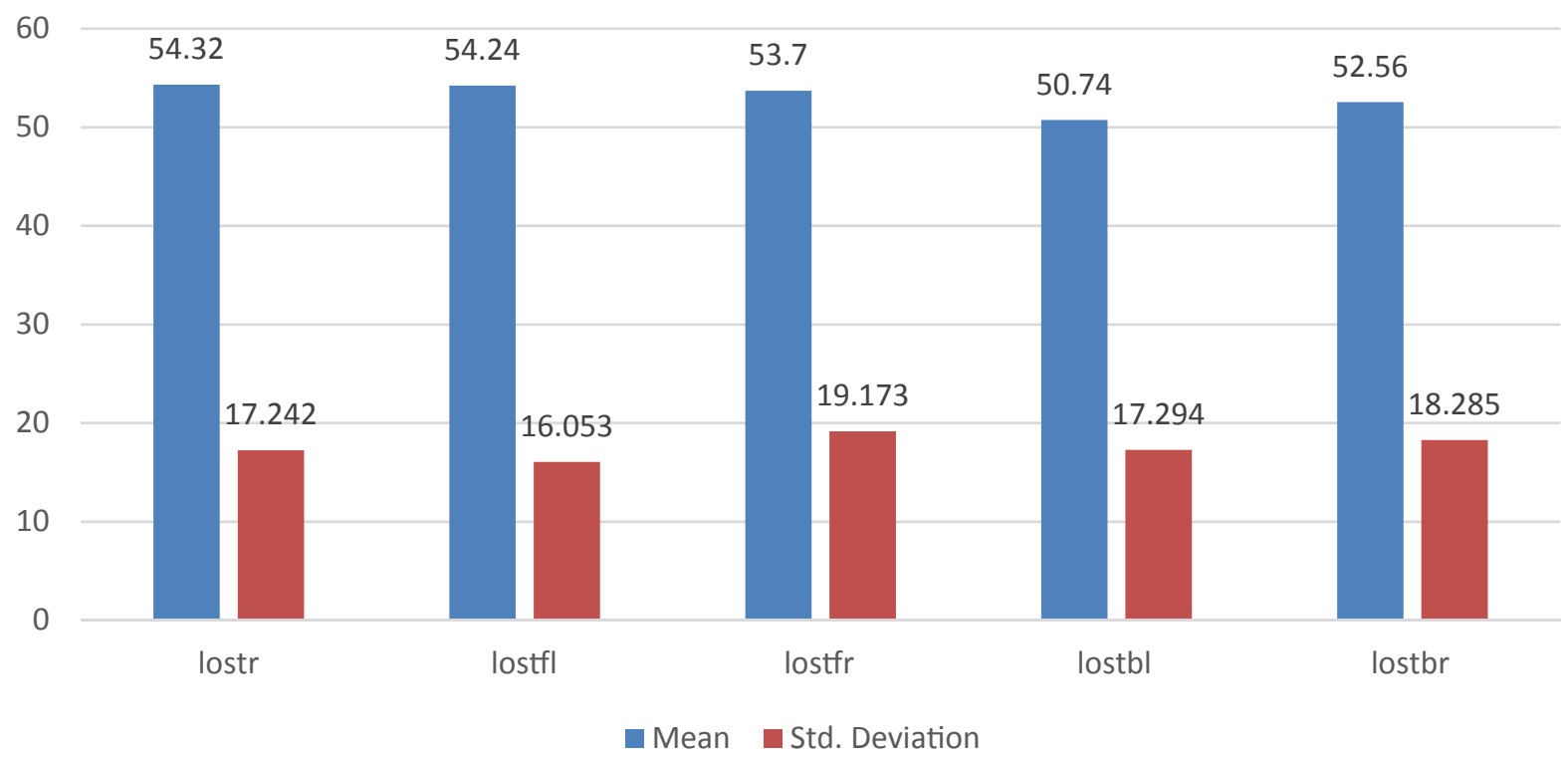

Figure 6. Explain the frequency distribution of limits of stability right (LOSTR) with mean $54.32 \pm 17.242$, limits of stability forward left (LOSTFL) with mean $54.24 \pm 16.053$, limits of stability forward right (LOSTFR) with mean $53.7 \pm 19.173$, limits of stability backward left (LOSTBL) with mean $50.74 \pm 17.294$ and limits of stability backward right (LOSTBR) with mean 52.56 \pm 18.285 .

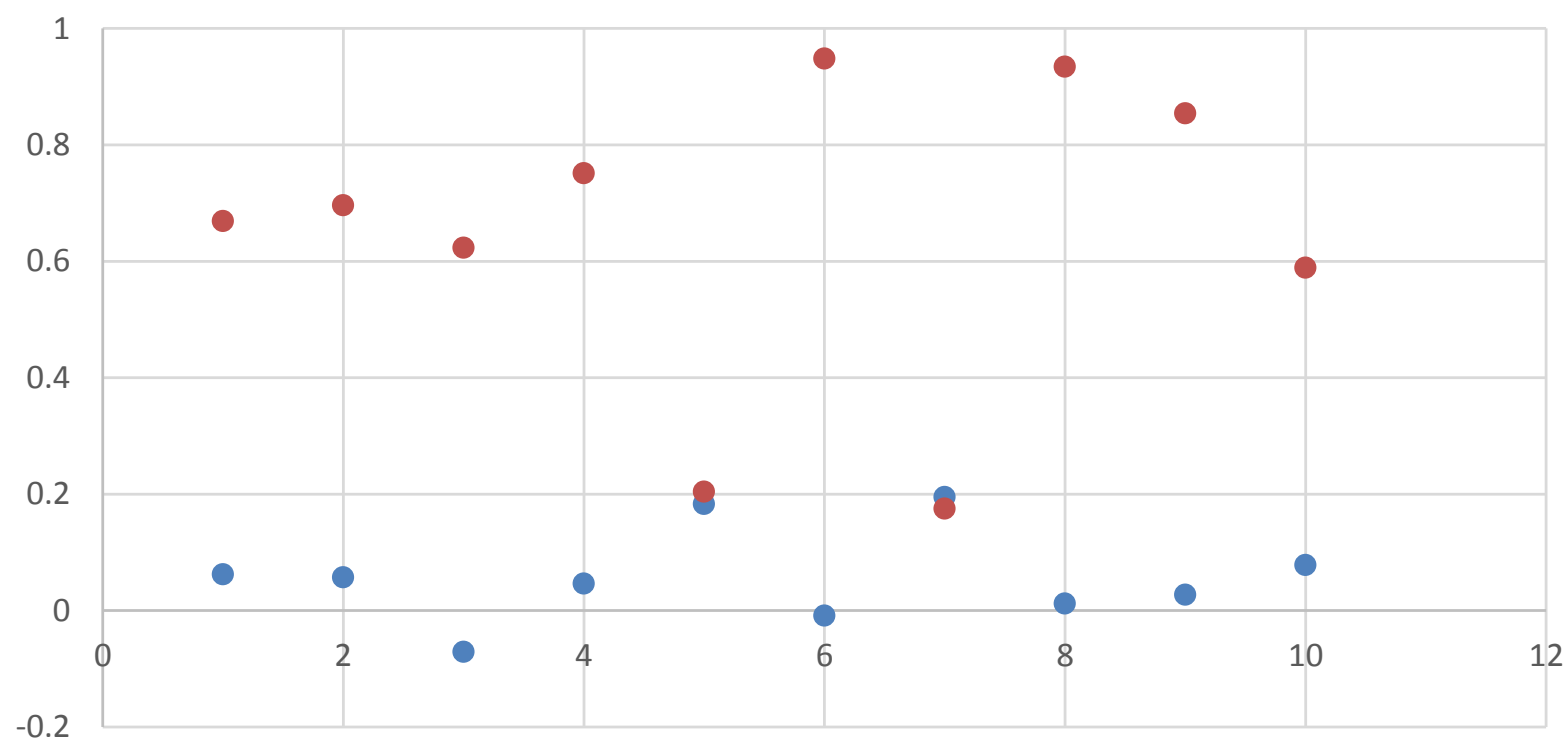

Figure 7. The person correlation between the BMI from one side and fall risk test demonstrated as (overall stability index), stability test demonstrated by (overall limits of stability, limits of stability in forward direction limits of stability in backward direction, limits of stability towards left side, limits of stability towards right side,towards forward to the left and to the right, towards backward to the left and to the right)and postural stability test demonstrated by (overall stability index, antero-posterior stability index, Medio lateral stability index) on the other side and it shows no correlation between BMI and all other parameters. 
Table 1. Represents the descriptive statistics of all parameters (Limits of Stability Right (LOSTR), Limits of Stability Forward Left (LOSTFL) Limits of Stability Forward Right (LOSTFR), Limits of Stability Backward Left (LOSTBL) Limits of Stability Backward Right (LOSTBR), Overall Stability (Overalls) Index Limits of Stability (LOSTO) and Limits of Stability Forward (LOSTF) Limits of Stability Backward (LOSTB) Limits of Stability Left (LOSTL).

\begin{tabular}{|c|c|c|c|}
\hline Variables & Number & Mean & Std. Deviation \\
\hline Age & 50 & 21.20 & 1.863 \\
\hline Height & 50 & 157.56 & 3.939 \\
\hline Weight & 50 & 85.55 & 7.824 \\
\hline BMI & 50 & 34.37 & 2.941 \\
\hline OVERALLSI & 50 & 2.27 & 1.056 \\
\hline LOSTO & 50 & 47.08 & 13.540 \\
\hline LOSTF & 50 & 58.72 & 15.305 \\
\hline LOSTB & 50 & 58.52 & 20.617 \\
\hline LOSTL & 50 & 60.54 & 16.511 \\
\hline LOSTR & 50 & 54.32 & 17.242 \\
\hline LOSTFL & 50 & 54.24 & 16.053 \\
\hline LOSTFR & 50 & 53.70 & 19.173 \\
\hline LOSTBL & 50 & 50.74 & 17.294 \\
\hline LOSTBR & 50 & 52.56 & 18.285 \\
\hline
\end{tabular}

Table 2. The Pearson correlation between the BMI from one side and fall risk test demonstrated as (overall stability index), stability test demonstrated by (overall limits of stability, limits of stability in forward direction limits of stability in backward direction, limits of stability towards left side, limits of stability towards right side, towards forward to the left and to the right, towards backward to the left and to the right)and postural stability test demonstrated by overall stability index, antero-posterior stability index, Medio lateral stability index) on the other side and it shows no correlation between BMI and all other parameters (Limits of Stability Right (LOSTR), Limits of Stability Forward Left (LOSTFL) Limits of Stability Forward Right (LOSTFR), Limits of Stability Backward Left (LOSTBL) Limits of Stability Backward Right (LOSTBR). Overall Stability (Overalls) Index Limits of Stability (LOSTO), Limits of Stability Forward (LOSTF) Limits of Stability Backward (LOSTB) Limits of Stability Left (LOSTL).

\begin{tabular}{|c|c|c|}
\hline \multirow{2}{*}{ Variables } & \multicolumn{2}{|c|}{ BMI } \\
\hline BMI & Pearson Correlation & Sig. (2-tailed) \\
\hline OVERALLSI & 1 & 1 \\
\hline LOSTO & 0.062 & 0.669 \\
\hline LOSTF & 0.057 & 0.696 \\
\hline LOSTB & -0.071 & 0.623 \\
\hline LOSTL & 0.046 & 0.751 \\
\hline LOSTR & 0.183 & 0.204 \\
\hline LOSTFL & -0.009 & 0.948 \\
\hline LOSTFR & 0.195 & 0.175 \\
\hline LOSTBL & 0.012 & 0.934 \\
\hline LOSTBR & 0.027 & 0.854 \\
\hline & 0.078 & 0.589 \\
\hline
\end{tabular}

\section{Conclusion}

BMI is not the only determent of balance but it may be of great concern if it is added to other factors, which affect balance.

\section{Limitations}

1. Sampling in the scope of the university and the lack of sufficient cases "old age ".

2. The Biodex is not available in large hospitals to measure the available cases there.
3. The lack of cooperation and approval to conduct tests in some cases.

\section{Recommendations}

1. Obese individual should be educated about the seriousness of the imbalance to avoid falling.

2. Obese individual should be educated to do exercises.

3. Should do this study in a large number of obese male and female.

\section{References}

1. World Health Organization. Obesity: Preventing and managing the global epidemic. WHO. 2000.

2. Bianchini F, Kaaks R, Vainio H. Overweight, obesity, and cancer risk. The Lancet Oncology. 2002;3:565-74.

3. James WPT, Jackson-Leach $\mathrm{R}$, Mhurchu $\mathrm{CN}$, et al. Overweight and obesity (high body mass index). In: Comparative quantification of health risks: Global and regional burden of disease attributable to selected major risk factors. WHO. 2004:497-596.

4. Yang P, Zhou Y, Chen B, et al. Overweight, obesity and gastric cancer risk: Results from a meta-analysis of cohort studies. Eur J Cancer. 2009;45:2867-73.

5. Freedland SJ, Wen $\mathrm{J}$, Wuerstle $\mathrm{M}$, et al. Obesity is a significant risk factor for prostate cancer at the time of biopsy. Urology. 2008;72:1102-05.

6. Nguyen NT, Magno CP, Lane KT, et al. Association of hypertension, diabetes, dyslipidemia, and metabolic syndrome with obesity: Findings from the National Health and Nutrition Examination Survey during 1999 to 2004. J Am Coll Surg. 2008;207:928-34.

7. Abbasi F, Brown BW, Lamendola C, et al. Relationship between obesity, insulin resistance, and coronary heart disease risk. J Am Coll Cardiol. 2002;40:937-43.

8. National Institutes of Health. Clinical guidelines on the identification, evaluation, and treatment of overweight and obesity in adults - The evidence report. Obes Res. 1998;2:51-209.

9. Pollock AS, Durward BR, Rowe PJ, et al. The effect of independent practice of motor tasks by stroke patients: a pilot randomized controlled trial. Clin Rehabil. 2002;16:473-80.

10. Blaszczyk JW, Lowe DL, Hansen PD. Ranges of postural stability and their changes in the elderly. Gait Posture. 1994;2:11-7.

11. Pollock AS, Durward BR, Rowe PJ, et al. What is balance? Clin Rehabil. 2000;14:402-06.

12. Robertson M, Devlin N, Scuffham P, et al. Economic evaluation of a community based exercise program to prevent falls. J. Epidemiol. 2001;55:600-06.

13. Reed-Jones RJ, Dorgo S, Hitchings MK, et al. WiiFit ${ }^{\mathrm{TM}}$ plus balance test scores for the assessment of balance and mobility in older adults. Gait Posture. 2012;36:430-33. 
Citation: Ibrahim AA, Mohamed AMR, Kamel EM, et al. The relevance of balance assessment to detect balance deficits in obese subjects: A cross sectional study. Biol Med Case Rep. 2018;2(1):15-20.

14. Janssen I, Katzmarzyk PT, Ross R. Body mass index, waist circumference and health risk: Evidence in support of current NationalInstitutes of Health guidelines. Arch Intern Med. 2002;162:2074-9.

15. kumaresan A, Kavithayini T. The impact of body mass index on performance of the star excursion balance test. Int J Pharm Sci and Health Care. 2012;2.

16. Ailton JO, Carneiro P, Adilton AO. et al. Obese elderly women exhibit low postural stability: A novel threedimensional evaluation system clinic. 2012;67:475-481.

\section{*Correspondence to:}

Ibrahim AA

Department of Physiotherapy

Cairo University Hospitals

Cairo

Egypt

Tel: +20223643524

E-mail: boodytwo48@gmail.com 